\title{
Prácticas de reapropiación de espacios comunitarios en el Cerro Cordillera. Un análisis etnográfico del Taller de Acción Comunitaria (TAC), la Población Obrera de la Unión y el Espacio Santa Ana, Valparaíso, Chile MAXIMILIANO SOTO
}

> Centro de Desarrollo Urbano Sustentable - CEDEUS, Pontificia Universidad Católica de Chile, Santiago, Chile. msotm@uc.cl NAHUEL QUIROGA CORVALÁN

$>$ Escuela de Arquitectura, Universidad Central, Santiago, Chile.nahuelquiroga@alumni.nd.edu MARCELO GONZÁLEZ CISTERNAS

> Comisión de Estudios del Espacio Santa Ana, Valparaíso, Chile. espaciosantaana@gmail.com

Universidad de Valparaíso

Facultad de Arquitectura

Revista Márgenes

Espacio Arte Sociedad

Prácticas de reapropiación de espacios comunitarios en el Cerro Cordillera. Un análisis etnográfico del Taller de Acción Comunitaria (TAC), la Población Obrera de la Unión y el Espacio Santa Ana, Valparaíso, Chile

Septiembre 2014 Vol $11 \mathrm{~N}^{\circ} 14$

Páginas 52 a 62

ISSN elec. 0719-4463

ISSN imp. 0718-4034

Recepción: mayo 2014

Aceptación: agosto 2014

RESUMEN

El presente artículo es el resultado de un análisis cualitativo de tipo etnográfico realizado en tres espacios del Cerro Cordillera, de Valparaíso, que se enmarcan dentro de prácticas de reapropiación socio-culturales registradas desde comienzo de la década de 1990: a. el Taller de Acción Comunitaria (TAC), b. la Población Obrera de La Unión y c. el Espacio Santa Ana. Tres espacios que reúnen experiencias diversas en lo individual, colectivo y comunitario -a modo de entornos de interacción-, haciendo visibles dinámicas intersubjetivas que adquieren una cierta singularidad al interior de éste cerro. Entornos donde los sujetos establecen rutinas que conforman su cotidianeidad, dentro de un proceso de construcción de un Nosotros cultural como unidad de sentido interaccional.

PALABRAS CLAVE

etnografías urbanas, reapropiaciones, espacios comunitarios, Cerro Cordillera, Valparaíso

Reappropriation of community spaces practices in Cordillera Hill. An ethnographic analysis of the Community Action Workshop, Workers Village in La Unión and Espacio Santa Ana, Valparaíso, Chile ABSTRACT

This article is the result of a qualitative ethnographic analysis conducted in three areas of the hill "Cerro CordiIlera", in Valparaíso, Chile: a. Action Workshop Community (TAC), b. Población Unión Obrera collective housing project, and c. Espacio Santa Ana. Three spaces that bring together diverse experiences in the individual, group and community -as an interaction environments- making visible intersubjective dynamics that acquire a certain uniqueness within this hill. Environments where subjects establish routines that make up their everyday lives, in a process of building a culturally and Us unit interactional sense.

KEYWORDS

urban ethnographies, re - appropriations, community spaces, Cerro Cordillera, Valparaíso

Pratiques de re-appropriation d'espaces communautaires dans la Colline Cordillera. Une analyse ethnographique de l'Atelier d'Action Communautaire (TAC), la Población Obrera de l'Union et l'Espace Santa Ana, Valpa-

raiso, Chili

RÉSUMÉ

Le présent article est le résultat d'une anlyse qualitative de type etnographique appliquée sur trois endroits du Cerro Cordillera, à Valparaíso, dans le cadre d'un processus de pratiques socio-culturelles de réappropiations. Des pratiques qui ont été enregistres dès le début des années 1990: a. l'Atelier d'Action Communautaire (TAC), 
b. la Población Obrera de la Unión y c. le Espacio Santa Ana. Trois espaces qui gardent des experiences divers entre l'individuel, le collectif et le communutaire -en tant qu'environnement d'interaction-, en mettant en lumière dynamiques intersubjectives qui devienent singulières à l'intérieur de la colline. Des environnements dont les sujets établissent des routines, qui donnent forme à leurs quotidiannéite, autour d'un processus de construction d'un Nous culturel, en tant qu'unité de sens interactionnelle.

MOTS CLÉS

etnographies urbaines, reappropiations, espaces communutaires, Cerro Cordillera, Valparaíso

\section{INTRODUCCIÓN}

L'espace urbain est le produit sédimenté d'intentionnalités multiples, concurrentes ou successives, qu'il est de l'histoire accumulée et réinterprétée (Grafmeyer, 1999:157).

Al interior de un espacio natural como es el caso de los cerros y quebradas de Valparaíso se pueden observar diferentes prácticas de apropiación que dejan huellas que se inscriben y que transforman dicho espacio en un territorio una vez que dichas prácticas adquieren la dimensión de formas sociales (Simmel, 1996), estructurándose desde lo individual y comunitario ${ }^{1}$. Territorialidades que responden también a ciclos de continuidad cuando los procesos sociales contextuales alteran las apropiaciones y las rutinizaciones que se ejercen, dando origen a desapropiaciones o desvalorizaciones que producen abandonos, por ejemplo, de inmuebles y de espacios de uso público, pero también desarticulaciones del tejido social, en el caso de sindicatos y/o asociaciones. Todo un proceso que desvaloriza el territorio pero que dependiendo de las dinámicas producidas por los actores que habitan el lugar, pueden volver a revalorizarse activando reapropiaciones en función de nuevas prácticas de identificación y reconocimiento de un territorio compartido.

Las diferentes prácticas de reapropiación, que producen territorio y que constituyen a la vez diferentes formas de conocimiento y de experticia, constituyen todo un campo de estudio que, desde un análisis de las intersubjetividades, permite una aproximación entre los estudios culturales y los estudios urbanos (Lindón, 2007). Una problemática de estudio que se puede observar e identificar en un espacio definido como lo es el Cerro Cordillera, en Valparaíso, responden a la presencia-acción de diferentes actores (públicos, privados, técnicos, habitantes, etc.). Todo un tejido relacional e interaccional que responde a posiciones, experiencias, valores e intereses diversos; donde las interacciones sociales dan origen a lo que denominamos "comunidad de actores" (Reynaud, 1989), las cuales son atravesadas por tensiones y conflictos, pues los actores no poseen los mismos intereses y valores, y tampoco comparten las mismas definiciones de sus prácticas.

Un terreno que ha sido abordado a través de un método etnográfico, apoyándose en registros fotográficos y en notas de campo ${ }^{2}$; donde prevalece el modo predominante de la moderna autoridad del trabajo de campo, "Estás allí... porque yo estuve allí" (Clifford, 1995:40). Un método cualitativo que nos ayudará a elaborar una primera tipología de diferentes niveles de conciencia y de valorización de tres entornos de interacción: el Taller de Acción Comunitaria (TAC), la Población Obrera de La Unión y el Espacio Santa Ana. Las etnografías registrarán recorridos al interior y exterior de estos lugares, identificando unidades de sentido asociadas a usos, prácticas y representaciones en la reapropiación de los mismos.

Hemos estructurado este artículo en tres partes. Una primera para explicar los entornos de interacción en función de niveles de conciencia y de reconocimiento (sección I). Enseguida una revisión socio-histórica de la ciudad de Valparaíso en general y del Cerro
Cordillera en particular (sección II); para finalizar con un análisis de las prácticas de reapropiación que se han observado e identificado en los tres espacios de interacción señalados (sección III).

\section{LOS ENTORNOS DE INTERACCIÓN COMO PRODUC- TORES DE INTERSUBJETIVIDADES: ENTRE TRANSAC- CIONES Y REGULACIONES SOCIALES}

En un principio hemos privilegiado un análisis sociológico. La elección de un análisis comparativo profundo ha implicado una apertura hacia la antropología y la historia social. Así hemos pasado a una aproximación socio-antropológica para integrar los particularismos (cross cultural) y una aproximación societal con el fin de establecer principios generales que expliquen los procesos de reapropiación desde su patrimonio inmaterial, en función de una comprensión de prácticas de reapropiación de entornos de interacción. El eje central es socio-antropológico y se desarrolla a partir de una relación entre la forma material de la vida social y sus formas simbólicas, sus representaciones y usos, y significados del espacio habitado.

Los entornos de interacción se conforman desde los diferentes lugares en que los sujetos establecen las rutinas que estructuran su vida cotidiana. La significación de éstos entornos se asocia a la construcción del "sentido común", el cual es descrito de acuerdo a lo que en el lenguaje de la fenomenología social se denomina mundo de vida (Schutz, 1972). Es éste concepto el que nos permite abordar el TAC, la Población Obrera de La Unión y el Espacio Santa Ana como formas urbanas/culturales (sistema simbólico de representación) y como formas urbanas/políticas (sistema de transacción y de producción social). Dos formas de análisis que se complementan, ayudando a avanzar en el análisis de lo barrial entendido en función de segregaciones y agregaciones: El barrio es -en estos términos- un indicador de la segregación en el uso del espacio urbano de determinados sectores sociales y, en consecuencia, lo que se distingue como parte de un todo (Gravano, 2005:13). Desde esta mirada del Cerro Cordillera podría estar conformado por varios barrios obreros que responden a una producción social, económica y simbólica específica. Para otros sujetos pueden ser espacios de ejercicio del poder, espacios controlados, en los cuales a través de la relación sujeto-territorio se recomponen identidades (Lindón, 2007:12). Sin embargo esta noción del espacio no da cuenta de las prácticas de apropiación que ejercen los individuos, sino que más bien de la posición que ocupan, apelando a integrar otros conceptos operatorios como territorio y territorialidad para ir develando los procesos que se articulan en la construcción de un Nosotros cultural.

Dans la mesure où la ville est un espace partagé, elle est un milieu de vie qui amène tout un chacun à "composer" avec autrui, et peut-être, du coup, avec soi-même (...) Les rapports de coopération, de concurrence ou de conflit différent selon la nature de ces enjeux: propriété $d u$ sol ou du bâti, appropriation matérielle ou symbolique des territoires urbains, protection de l'intimité domestique ou 
de l'entre-soi social, accès aux services collectifs ou aux espaces publics (Grafmeyer, 1999:159).

En las prácticas de inscripción territorial el actor social se define por una tensión entre lo individual y lo colectivo, siguiendo dos ejes opuestos: subjetividad/intersubjetividad y reconocimiento/desconocimiento, o conocimiento deformado. El esquema 1 ilustra estas oposiciones: de un lado la conciencia subjetiva e intersubjetiva, que se explica por una tensión entre identidad y alteridad; de otro lado, el reconocimiento y desconocimiento del patrimonio inmaterial, que se explica por una tensión entre lo desconocido individual y lo conocido socialmente.

\begin{tabular}{|c|c|c|}
\hline & RECONOCIMIENTO & \\
\hline & \multirow{4}{*}{4} & \\
\hline $\begin{array}{l}\text { Conocido/ } \\
\text { individual }\end{array}$ & & $\begin{array}{l}\text { Conocido/ } \\
\text { colectivo }\end{array}$ \\
\hline Subjetivo & & Intersubjetivo \\
\hline \multirow[t]{2}{*}{$\begin{array}{l}\text { Desconocido/ } \\
\text { individual }\end{array}$} & & $\begin{array}{l}\text { Desconocido/ } \\
\text { colectivo }\end{array}$ \\
\hline & DESCONOCIMIENTO & \\
\hline
\end{tabular}

$>$ Esquema 1. Niveles de conciencia en los entornos de interacción y en las prácticas de reapropiación

Los niveles de conciencia en el conocimiento de lugares, ya sean entornos de interacción o espacios identitarios, producen una tensión (Ver Esquema 1.) entre la conciencia subjetiva e individual de un lado, y la conciencia intersubjetiva y colectiva, de otro lado. El reconocimiento es definido como la extensión de la conciencia individual $^{3}$. Existen desfases entre el reconocimiento de una vieja construcción por los expertos que estudian el inmueble para declararlo histórico y, por otro lado, el reconocimiento de su valor por el individuo que lo habita. Se pueden identificar dos tipos de reconocimiento entre la experticia erudita y la experticia profana, donde los elementos que el arquitecto reconoce y valoriza no son los mismos que los que pueden ser reconocidos y revalorizados por los residentes y/o habitantes. Este paso o extensión permite la construcción del individuo como actor, miembro de una "comunidad patrimonial”. Una comunidad que reagrupa a aquellos que están de acuerdo con la defensa de un bien patrimonial, pese a tener manifiestas oposiciones, constituyendo un conjunto de regulaciones conjuntas puntuales (Reynaud, 1979). Una dinámica social que se puede visualizar en el caso de los ascensores de Valparaíso que son monumentos históricos pero también medios de transporte necesarios para aquellos que habitan en los cerros, o por lo menos en la primera cota de éstos (Soto, 2014b).

Se puede abordar, por ejemplo, la puesta en valor de un inmueble a partir de representaciones y discursos de actores sociales: el agente de turismo, el urbanista, el político, el conservador de museos, el agente inmobiliario, etc. Un proceso que requiere una regulación conjunta para llegar a un compromiso resultante de reglas aceptadas por la multiplicidad de actores, constituyendo un conjunto razonablemente coherente (Reynaud, 1979). El concepto de regulación conjunta puede responder a diferentes niveles de decisión: la junta de vecinos, el municipio, la asociación de copropietarios de la Población de la Unión Obrera o el TAC. La regulación conjunta se sitúa entre la decisión individual y la regla colectiva, cuando las oposiciones de los actores sociales estructuran las parejas de tensiones, como si fuera un juego colectivo. Por ejemplo, en la regulación de la recuperación de una quebrada, se pueden identificar parejas de tensión que se oponen entre la dimensión material del espacio natural y la inmaterialidad que permanece en las representaciones sociales de los diversos usuarios/usos que le son asociados (Ver Esquema 2).

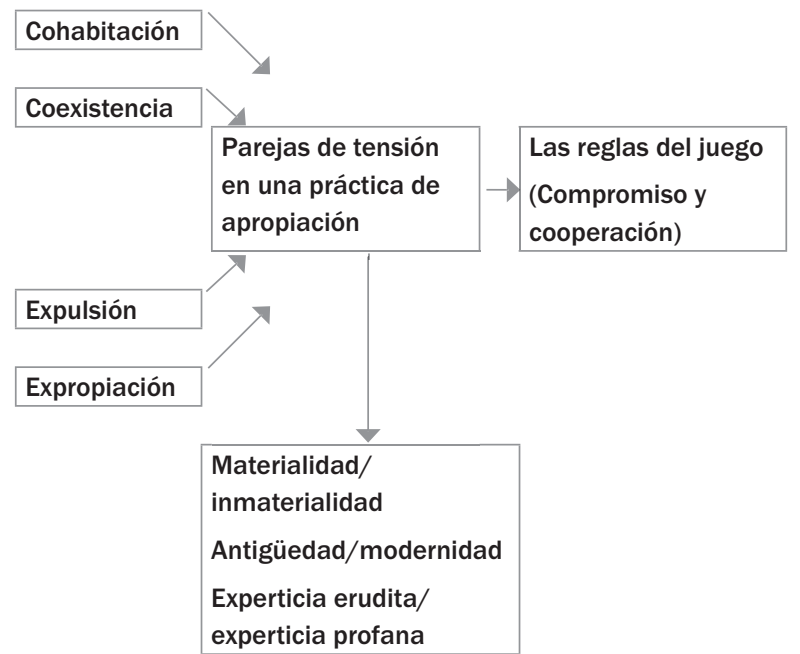

$>$ Esquema 2. Visión parcial del origen de las reglas en la regulación de una práctica de apropiación

La reapropiación de entornos de interacción de valor identitario, tiene un impacto económico, ligado a los modos de vida de los nuevos habitantes con una mayor capacidad de consumo y nuevas formas de interacción social entre transacciones y regulaciones sociales en la búsqueda de compromisos prácticos. En este contexto, las transacciones sociales y las regulaciones conjuntas no piensan en un contrato social definitivo, pues la renegociación es permanente. Estas dos vertientes teóricas se focalizan en el compromiso práctico para llegar a acuerdos en situaciones conflictuales. La regulación conjunta prefiere hablar de solución, pese a que el concepto de compromiso está presente como acuerdo provisorio. Por el contrario la transacción social aborda el compromiso práctico como una de las nociones centrales dando un espacio al disenso.

La estructura de la teoría sociológica, los paradigmas de la transacción social (Remy et al., 1991 [1978]) y la regulación conjunta (Reynaud, 1989) permiten abordar una nueva mirada sobre las formas sociales emergentes en el contexto de la puesta en valor de bienes patrimoniales, como es el caso del TAC o del Espacio Santa Ana, que generalmente van acompañados de conflictos de reapropiación y de interpretación de valores culturales. El conflicto interviene en la revalorización: entre la materialidad y la inmaterialidad, que están presentes al interior del discurso y de las representaciones de sitios. Esta tensión se articula con dispositivos que funcionan como reguladores y productores de vínculos sociales, donde la presencia de conflictos contribuye en la producción de nuevas formas sociales. Elaborar compromisos prácticos para resolver conflictos, que ligan estrictamente el uso y el valor, es el resultado de transacciones, negociaciones y regulaciones.

El análisis de las relaciones sociales, y sobre todo de prácticas de reapropiación en un contexto de revalorización, comienza por una conexión entre la forma urbana y la forma social. Una nueva configuración se produce en los procesos transaccionales de apropiaciones, desapropiaciones y reapropiaciones. De esta manera las transacciones sociales integran el reconocimiento de los habitantes y de sus espacios, pues ellas analizan la relación social en el ejercicio cotidiano, a la vez como inductor y como inductivo en la 
producción del territorio (Remy, 1992:89); donde los habitantes, en un sentido amplio, articulan sus experiencias contenidas desde una apropiación ya inscrita en el territorio, y nuevas prácticas en función de miradas y recorridos nuevos sobre el territorio reapropiado $\mathrm{y}$, a la vez, resimbolizado.

\section{UNA REVISIÓN SOCIO-HISTÓRICA DE VALPARAÍSO Y DEL CERRO CORDILLERA}

Valparaíso es conocido como el principal puerto de Chile, un puerto mítico donde cohabita el plan y los cerros. Para algunos es la ciudad de los cuarenta y cuatros cerros (Sáez, 2001). Una ciudadPuerto que es testimonio del desarrollo industrial y comercial de una época, que configura un tejido socio-urbano que responde a asentamientos particulares posibles de distinguir, por ejemplo, en el Cerro Cordillera. Un proceso que significó apropiaciones de quebradas y de terrenos en alturas para muchos campesinos que venían del valle central buscando nuevas oportunidades laborales y que estaban acostumbrados a vivir en terrenos llanos. Los cerros con sus quebradas y hondonadas que los separan conforman límites al habitar, a las urbanizaciones. La complejidad consiste en imaginar que los cerros fueron islas que iban a la deriva por los mares hasta venir a encallar en Valparaíso (Cruz, 2003:218).

Una vez finalizado el proceso de independencia de Chile, y el consecutivo monopolio económico con España en 1818, numerosas casas comerciales inglesas se instalan en Valparaíso, activando un crecimiento comercial. En esta actividad, los comerciantes ingleses son privilegiados pues entre un $30 \%$ y un $60 \%$ de todos los productos chilenos eran exportados a Inglaterra, y en su mayoría se embarcaban en Valparaíso. En este contexto los ingleses representaban la "última diáspora comercial" a una escala del desarrollo de corporaciones multinacionales modernas (Cavieres, 1986). Este desarrollo de actividades comerciales repercute sobre la transformación de la morfología social, donde las actividades y su respectiva evolución, estructurada por ciclos, permite distinguir entre una arqueo-burguesía, una nueva burguesía y una pequeña burguesía (Ledrut, 1976).

El control de las ventas por parte de la Asociación de Productores de Salitre reforzó el liderazgo de Valparaíso en este rubro fundamental para la economía chilena. Sin embargo, en la medida que los crecientes problemas de la industria salitrera afectaban los ingresos fiscales, la injerencia del gobierno se hizo más marcada. La decisión del controvertido Ministro de Hacienda Pablo Ramírez de terminar con la centralización de las ventas y promover el apoyo estatal a la industria a raíz de la crisis de 1929, desplazaba el centro de decisiones hacia Santiago. La Superintendencia de salitre y Yodo, creada al año siguiente, tenía entre sus objetivos vigilar las utilidades de los intermediarios en el negocio salitrero lo que afectaba derechamente al mundo comercial porteño (Couyoumdjian, 2000:93).

En el siglo XIX, la clase emergente de Chile constituye una aristocracia del dinero, ya sea por herencia, o ya sea por las fortunas adquiridas producto de las actividades comerciales y mineras de la época. La nueva burguesía emergente de la época, comienza a ser asimilada por la aristocracia colonial terrateniente, mayoritariamente concentrada en Santiago (Coujoumdjian, 2000). El perfil profesional se resume a sus funciones de propietarios de pequeñas tiendas y casas comerciales, y a funciones concernientes a servicios públicos, de ingenieros y de suboficiales de la Armada.
Por otro lado a mediados del siglo XIX Valparaíso comienza a experimentar un visible crecimiento demográfico. Para 1819, a pocos meses de haberse firmado la declaración de Independencia de Chile, Valparaíso contaba con una población de 5.000 habitantes; población que para 1885 llega a 122.500 individuos (Benavides et al., 1994; Calderón, 1986; Sáez, 2001). En 66 años la población de Valparaíso experimentó un crecimiento demográfico record de $1,5 \%$ por año. El crecimiento coincide con el desarrollo urbano de la ciudad. El período 1860-1880, por ejemplo, corresponde, según las palabras de Alfonso Calderón (1986), a la edad de oro de la ciudad en relación al desarrollo de actividades innovantes, a las obras de abovedamiento de canales y de higienización de la ciudad, la construcción y la instalación de equipamientos urbanos: correo central, trenes, ascensores, plazas y parques, centros comerciales y una serie de inmuebles tanto públicos como privados (Couyoumdjian, 2000).

Proceso que significó la producción de nuevas prácticas asociadas a nuevos desplazamientos, ritmos y horarios; configurando un sentido cotidiano particular del espacio para sujetos que veían por primera vez el mar sin nunca antes haber observado flujos comerciales de tanta envergadura como eran los que se podían ver a fines del siglo XIX en Valparaíso. Claramente un nivel de industrialización que es posible visualizar y testimoniar en, por ejemplo, los ascensores. A fines del siglo XIX, los ascensores eran un moderno medio de transporte que conectaba el plan de la ciudad con la primera cota donde se ubicaban las residencias de aquellas familias inmigrantes que habían logrado amasar una importante fortuna a escala local, como es el caso de los Gervasoni, Baburizza, Zanetti, entre otros. Familias que se instalaron en cerros cercanos al barrio puerto, como es el caso del Cerro Cordillera, y del barrio financiero como el Cerro Concepción y Alegre. Los ascensores son un reflejo del crecimiento económico de Valparaíso que, con 150.000 habitantes en 1900, juega un rol fundamental en el comercio internacional y en el desarrollo económico de la ciudad-puerto ${ }^{4}$.

A fines del siglo XIX la estructura urbana de Valparaíso se caracterizaba por la presencia de cinco sectores claramente definidos: el Puerto donde se localizaban las actividades comerciales; los cerros Cordillera, Alegre y Concepción que correspondían a los principales sectores residenciales de la arqueo-burguesía; el Almendral, barrios residencial y comercial situado en el plano; el Cerro Barón, otro sector residencial, y finalmente, Playa Ancha, sectores residenciales para los obreros y para la "clase media" emergente (Castillo, 2005).

Sin embargo a comienzo del siglo XX, la historia de la ciudad y de su desarrollo se vieron fuertemente afectados por el terremoto de 1906 y la apertura del Canal de Panamá en 1914. Particularmente con el sismo la ciudad perdió el $2 \%$ de su población; $85 \%$ de las manzanas ubicadas principalmente en el Almendral fueron destruidas, produciendo una consecutiva movilidad residencial desde los cerros hacia Viña del Mar, la ciudad más próxima del sector y que resistió de mejor manera el terremoto. Por otro lado la apertura del Canal de Panamá puso a término la edad de oro de la Ciudad-Puerto, reduciendo de manera considerable el paso de embarcaciones por el Estrecho de Magallanes y los arribos a Valparaíso. Dos acontecimientos que han debilitado la capital financiera, comercial y económica de Chile, transformando la Ciudad-Puerto en un lieu de villégiature très couru, mais aussi le lieu d'habitation d'une partie de la population de Valparaíso (parmi les couches moyennes et supérieures), contribuant de la sorte à accentuer le déclin du grand port (Matas, 2003:31). Esta movilidad residencial de la an- 
tigua burguesía de origen inglés, alemán e italiano, deja el lugar a sectores populares que se apropian de varios inmuebles del lugar. Es el caso, por ejemplo, de los cerros Cordillera y Santo Domingo, dos antiguos sectores emergentes próximos al Barrio Puerto que se desvalorizan y, a la vez, reorganizan en función de otros grupos sociales que comienzan a ocupar inmuebles abandonados y a ocupar nuevos sectores del cerro asociados a las quebradas de San Francisco y de José Tomás Ramos.

Después de la crisis económica que se inicia con el jueves negro de 1929 y que se deja sentir durante toda las décadas de 1930 y 1940 , y sobre todo en la década de 1950, el traslado de numerosas industrias produce un déficit económico en Valparaíso, que se acompaña de un aumento en el desempleo, una baja calidad de los servicios y una desvalorización del tejido urbano y social (Pinto, 1959).

Actualmente, y en el marco de todo un proceso de patrimonialización que viene experimentando la ciudad desde la declaración UNESCO del 2003, aún es posible diferenciar los cerros turistificados próximos al "antiguo" centro financiero como es el caso de los cerros Alegre y Concepción, de aquellos cerros populares y deteriorados próximos al "antiguo" centro portuario, o Barrio Puerto, como es el caso de los cerros Cordillera y Santo Domingo.

El Cerro Cordillera, particularmente, se localiza en el sector sur poniente de Valparaíso. Su nombre responde a las accidentadas características topográficas que presenta, con una acentuada pendiente y pocas planicies que dificultan la construcción. Está rodeado por dos quebradas, al norte la quebrada de José Tomás Ramos que lo separa del Cerro Alegre, y al sur la quebrada de San Francisco que lo separa del Cerro Toro. Sus primeros asentamientos se localizan en torno a la Plaza Eleuterio Ramírez que corresponde a la parte baja del cerro. Al caminar por el Cerro Cordillera es posible identificar una carga histórico-cultural en la que estamos inmersos, no sólo desde la institucionalidad, sino que también desde lo local, lo rutinario e inmediato, la configuración del espacio se articula, entre lo patrimonial, como historia oficial, y las singularidades socio-culturales del lugar.

Respecto al comercio microlocal propio del Cerro, identificamos que existen la presencia de una variedad importante de locales como lo son carnicerías, una ferretería, minimarkets, botillerías, entre otros negocios; muchas de las cuales son llamadas "Menestras". Todos ellos dentro del comercio a escala microeconómica que no da espacio a un comercio en escala macroeconómica, configurando un tejido de prácticas de interacción que da espacio a significaciones comerciales singulares del Cerro.

La vida comunitaria del Cerro Cordillera se concentra en ciertos espacios de interacción como son los clubes deportivos que realizan una serie de actividades en el espacio público, pese que para ciertos informantes la actividad de dichos clubes no es lo misma que antes, signando dicha práctica como algo que está en retirada sin mayor importancia. Es el caso del club Meteoro ubicado a un costado del Auditorio Guillermo Bravo y a escasos metros de la Población Gutemberg.

Este cerro siempre ha sido un cerro de harta lucha, anteriormente los clubes deportivos por ejemplo, hacian sus carnavales, todos los años, elegían reina, y hacían carros alegóricos, era muy bonito... el Guillermo Bravo se ocupaba para marchas, para hacer presentaciones, era precioso... y la gente en ese aspecto coopera, en ese aspecto la gente es unida. Además aquí por ejemplo a todos nos corresponde la Iglesia del Perpetuo del Socorro, todos pertenecemos a esa iglesia, entonces nos vemos, seguido... y bueno mis hijos participaban, los hijos de la vecina, el del frente, el de allá, entonces fue algo que de por sí, nos fuimos más uniendo, y eso sí que se ha perdido un poco, pero queda... es cuestión no más de que uno trate de incentivar a la gente y la gente responde en ese aspecto (Entrevista a Sra. Inés, 15.03.2014).

Se destacan también las actividades desarrolladas por el TAC y el Espacio Santa Ana donde los conceptos de autoconstrucción, autogestión y autorganización son viables, expresándose en actividades de recreación como talleres de autocultivo, pintura mural o reciclaje; pero también en actividades de comida solidaria, educación y capacitación, como es el caso de la actividad de autoconstrucción de ladrillos de adobe organizada por el Espacio Santa Ana en febrero 2014.

El Cerro Cordillera es próximo al centro histórico de Valparaíso, ubicándose entre una zona valorizada y una desvalorizada. Un cerro que conserva una imagen de barrio obrero, pese a los trabajos de rehabilitación-restauración que han afectado tanto el tejido urbano como social del lugar. El Cerro Cordillera es uno de los primeros cerros que se habitaron en Valparaíso, un poco más antiguo, y eso lo tiñe de algo distinto a los otros (...) tiene un patrimonio construido relativamente homogéneo (Juan Mastrantonio, Presidente del Colegio de Arquitectos de Valparaíso, entrevista del 31.07.2008). De hecho el sector bajo de este cerro está valorizado por el hecho de ser zona típica, pese a contener inmuebles que responden a diferentes períodos de construcción, como es el caso del antiguo taller de manufactura La Igualdad (Soto, 2014a), Ios conventillos de la calle Merlet, las casas rehabilitadas en la calle Víctor Hugo o el Museo Lord Cochrane.

El Cerro Cordillera es una zona donde originalmente se instalaron campesinos y obreros productos del movimiento migratorio campociudad y norte-centro, consecutivamente, atraídos por las actividades comerciales e industriales de la ciudad-Puerto (Salazar, 1985); dando origen en muchas ocasiones a instalaciones espontáneas en quebradas y terrenos sin urbanización y de difícil acceso.

\section{ANÁLISIS DE PRÁCTICAS DE REAPROPIACIÓN EN TRES ENTORNOS DEL CERRO CORDILLERA: TAC, POBLACIÓN OBRERA DE LA UNIÓN Y EL ESPACIO SANTA ANA}

El primer entorno de interacción estudiado corresponde al Taller de Acción Comunitaria (TAC). Esta organización comunitaria comenzó a trabajar en el Cerro Cordillera en 1988 y ha perdurado por más de dos décadas, ha promovido la idea del voluntariado, la asociatividad y la participación en la comunidad. EI TAC ha sabido promover el empoderamiento del ciudadano, donde éste se presenta como creador y partícipe de su realidad, una lógica que se contrapone con el perfil asistencialista existente a fines de la década de 1980.

Nosotros llegamos hace veinte años atrás, y puede ser medio autorreferente, acá donde estamos sentados que era un gran basural y a trabajar con niños sin ninguna otra perspectiva que esa; generar un espacio para los niños, un espacio con dignidad, un espacio que no fuera entregado por la Municipalidad, que no fuera la típica reivindicación del alcalde que venga a limpiar el basural, que venga el alcalde a no sé qué o que venga alguien a no sé qué. 
Sino que la propuesta nuestra era cómo poder generar ciudadanos que fueran capaces de tener propuesta, de pensar su barrio y de cambiar su barrio... (Patricia Castillo, Directora del TAC, entrevista del 18.07.2008).

A lo largo del tiempo, esta organización también ha funcionado como interlocutor entre las autoridades del gobierno regional, local, y otras organizaciones no gubernamentales. El TAC también ha participado en la creación de redes comunitarias a través de distintas organizaciones existentes en el Cerro Cordillera y otros sectores de Valparaíso.

Desarrollar un espacio de encuentro, reflexión, trabajo educativo, alegría, coordinación, trabajos comunitarios, sueños, expresión artístico-cultural, animación del barrio. Incentivar la construcción de conocimiento e implementación de nuevos métodos, en forma permanente, respetuosos de la vida cotidiana del barrio de manera coherente y armónica (Correa, 2013:33).

La idea de recuperación de la historia del cerro se enmarca dentro de este mismo discurso, lo que hace pensar que hay una re-significación de los contenidos, sean estos materiales, inmateriales, simbólicos, discursivos, entre otros. Una situación concreta que nos hace plantearnos varias interrogantes: Cuáles son los elementos de cambio y permanencia que se pueden constatar. Bajo qué lógica se inscriben, si tienen elementos de cruce y/o siguen una lógica lineal y ascendente o todo lo contrario. Qué sucede con las distintas generaciones y las distintas organizaciones presentes en el cerro, cuáles son las consecuencias de esta lógica sobre éstas, si permanecen producto de lo anterior o han contribuido a darle un nuevo significado.

Por otro lado, qué sucede con las nuevas prácticas y discursos que se generan producto de otras dinámicas asociadas, por ejemplo, a la globalización. Entender el espacio como un entorno dinámico, es decir, en permanente articulación con lo viejo y lo nuevo, con antiguas prácticas y nuevos desafíos, puede ser una manera de abordar el análisis, entendiendo que éste no es un entorno aislado, sino que en contacto con estructuras que activan prácticas de reapropiación del entorno.

Formamos comité en conjunto con la directiva, trabajamos y conseguimos el agua para calle cinco y aquí el alcantarillado, para todas las familias pa 'adentro... ese fue el primer paso que dimos, y bueno, y arreglamos nosotros esa pasada... no se podía pasar, porque no había pasada, no había nada... después fui haciéndome más conocida y llegué al TAC (...) Para mí el TAC, se lo digo con harta honradez, a pesar de que ya era una mujer ya con mis años, pero ahi para mí fue una escuela, porque yo anteriormente también tiraba cosas a la quebrada, ignorante, sin saber que hacía un daño, pero cuando llegué al TAC ahí como, le vuelvo a repetir, para mí fue una escuela porque aprendí bastante, ahí me enseñaron a muchas cosas y tuve roces con mucha gente de distinta índoles, y eso me sirvió mucho (...) La tía Paty para mí fue para mí como una profesora porque de ella aprendí bastante... ahí me fui terminando más de formar, y desde ese entonces aprendí a hacer proyectos también, y ahí fue cuando conquistamos esta plaza... hicimos unas terrazas para plantar, plantábamos de toda clase de verduras, y las repartíamos dentro de la comunidad (Entrevista a la Sra. Inés, 15.03.2014).

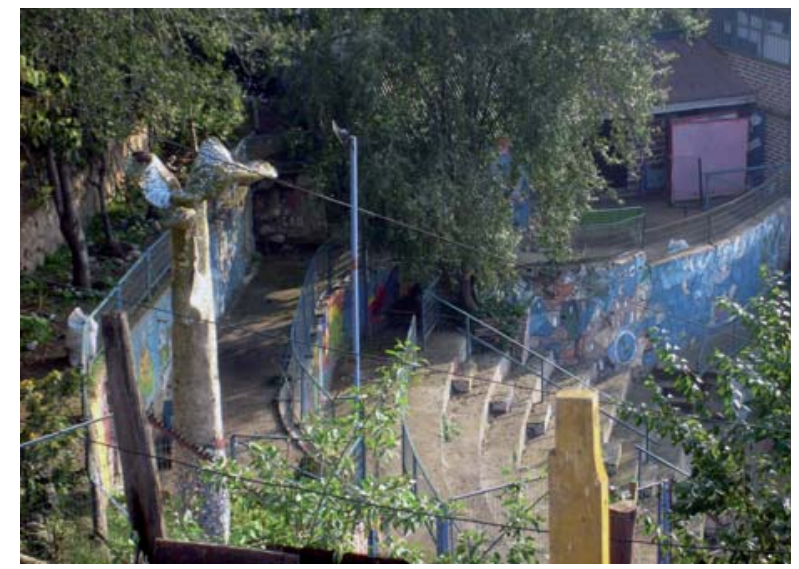

$>$ Figura 1. El Taller de Acción Comunitaria (TAC). Fotografía de M. Soto, agosto 2008

El TAC corresponde a una acción colectiva donde se realizan trabajos sociales desarrollándose como espacio de encuentro, reflexión, trabajo educativo, trabajos comunitarios, etc. Una de estas instancias de encuentro es la Biblioteca del TAC que se encuentra en la Población Gutemberg ubicada en el mismo Cerro Cordillera. Es una antigua casona donde se pueden observar ciertos mensajes, que a modo de signos de apropiación del entorno, expresan objetivos comunitarios: No es terrorismo, es resistencia; Ni por la razón ni por la fuerza o La tierra no tiene dueño.

Hay un reconocimiento de sentirse parte de una interacción dentro de un proceso compartido (Ricœur, 2000), como sucede entre el maestro y el discípulo cuando se dan cuenta del rol y de la posición que ocupan en la interacción; pero también en la dinámica donde cada uno cuenta con su competencia y donde el discípulo puede llegar a superar al maestro dependiendo de la situación concreta que responde a un contexto dado. Sin embargo hay un intercambio de roles, intereses, valores y prácticas.

El principal objetivo del TAC es comenzar un trabajo para aunar en la confianza de los habitantes del Cerro Cordillera y de todas las organizaciones locales que existen en este cerro (Patricia Castillo, directora del TAC, entrevista del 18.07.2008). La parte alta del cerro se caracteriza por la presencia de sectores populares que habitan desde años el lugar, muchos de ellos han pasado por el TAC, apropiándose y revalorizando los espacios de uso público como quebradas, plazas y calles. Uno de los primeros resultados de las acciones emprendidas fue la creación de una comisión para "recuperar" y rehabilitar La Población Obrera de La Unión; una acción que nació de la propia iniciativa de jóvenes que se movilizaron para mejorar la calidad de vida comunitaria.

Es así como un segundo entorno de interacción estudiado corresponde a la rehabilitación de la Población Obrera de La Unión, un viejo conventillo, que ha originado un proceso de revalorización acompañado de nuevas formas de apropiación de este patrimonio industrial. Este inmueble fue construido en 1870 por el arquitecto masón Carlos Lorca para dar hospedaje a familias campesinas que estaban llegando a Valparaíso; fenómeno que responde a los movimientos migratorios entre el campo y la ciudad del siglo XIX (Salazar, 1985).

En 1894, este inmueble fue comprado por una de las mayores fortunas de la época, la Sra. Juana Ross de Edwards, con el fin de conceder la propiedad del edificio a aquellas familias obreras organizadas en la Sociedad de Orden y Trabajo (Ferrada \& Jiménez, 2007). La administración quedó bajo la responsabilidad de 
un consejo de habitantes y del obispo de Valparaíso hasta 1950, cuando los arrendatarios comienzan a ocuparse ellos mismos de la administración del edificio sin hacer suficientemente atención a los trabajos de mantención.

Es así como la Unión Obrera comienza a desvalorizarse poco a poco, lo que explica en parte las acciones de desocupación emprendidas por la Municipalidad a lo largo de los años 1970, 1980 y 1990. Después de la dictadura militar (1990), una serie de organizaciones no gubernamentales (ONG) se instalan a lo largo del territorio nacional para reactivar las acciones sociales ligadas a la participación ciudadana, a la recuperación de espacios públicos, y a la economía local. Es así como la ONG Centro de Estudios, Capacitación y Asesoría Poblacional (CECAP) se instala en el Cerro Cordillera y rápidamente se transforma en un taller de acción social siguiendo los métodos elaborados por Paulo Freire, para transformarse en el Taller de Acción Comunitaria (TAC).

La Población Obrera de La Unión abarca una superficie de 3.263 $\mathrm{m}^{2}$, con un patio interior que armoniza toda la construcción de 34 departamentos distribuidos en tres pisos y conectados por pasillos interiores. Las 34 familias que albergan en su interior, un total de 120 personas, negociaron y acompañaron los trabajos de rehabilitación entre los años 2002 y 2008 junto al Ministerio de Vivienda y Urbanismo (MINVU), al Programa de Recuperación y Desarrollo Urbano de Valparaíso (PRDUV) y a la ONG española Junta de Andalucía.

El lugar hoy en día cuenta con la recuperación completa del edificio, donde muchas de las familias pudieron retornar a sus viviendas luego de años trabajando con vecinos, dirigentes y otros organismos para la rehabilitación del inmueble. Este trabajo permitió la integración social para mejorar la calidad de vida de las personas y así poder sacar a la luz la historia y la identidad del espacio y

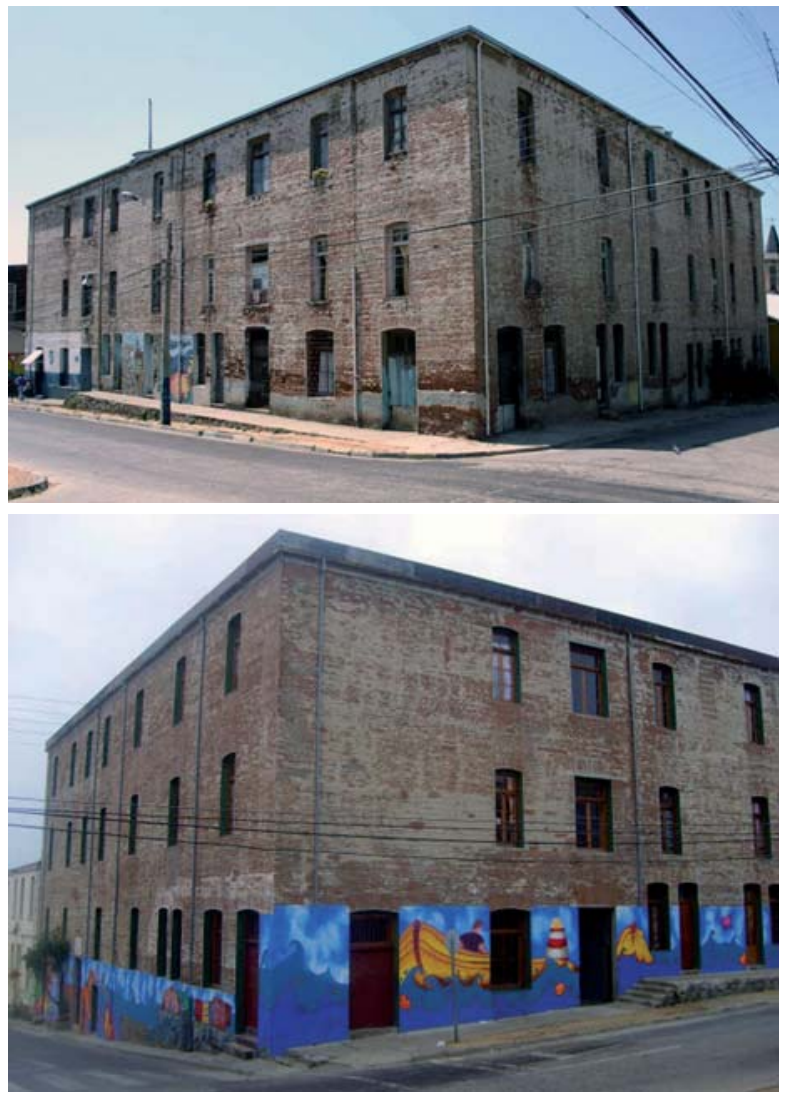

sus habitantes. Aquellos residentes que en algún momento fueron reacios a relacionarse con las personas que no eran propiamente de la Población, entendieron que a través del trabajo comunitario podrían construir una relación de fuerzas que permitiría la negociación con actores sociales institucionales para la recuperación de la construcción. Pero antes de este proceso era necesario que existiera un proceso de concienciación para trabajar la identidad de los actores permitiendo así la identificación colectiva de los miembros del grupo, lo que traería más adelante la organización, donde la cooperación de los actores sería la clave para el proceso de movilización (Dumas y Seguiré, 1997).

El tercer entorno de interacción estudiado corresponde al Espacio Santa Ana. Una ex capilla ubicada en la calle Balmes, detrás del inmueble de la Población Obrera de La Unión. Al acercarse se escuchan sonidos de martillos y herramientas, como también música de distintos estilos y ritmos. En este sitio se da lugar a la organización comunitaria Espacio Santa Ana que ocupa las dependencias de la ex capilla Santa Ana y del Hogar de Niñas María Goretti, un edificio de dos pisos. La ex capilla es del año 1886, y se construyó con el objetivo de colaborar con las labores eclesiásticas de la Iglesia La Matriz, pero fue cerrada en el año 1960 a toda la comunidad. En el año 2008 algunos habitantes organizados del Cerro Cordillera, muchos de los cuales fueron capaces de recuperar y rehabilitar la Población Obrera de La Unión, idearon una estrategia para restaurar el espacio en beneficio de ésta, desde una lógica de "autogestión comunitaria" que es difícil de entender para algunos actores del Cerro Cordillera que tienen otras formas de acción.

El Santa Ana, yo no sé, porque yo no estoy yendo a asambleas ni pertenezco a la cosa, yo no sé qué están discutiendo, no sé. Yo voy afuera a algunas cosas que me invitan, voy
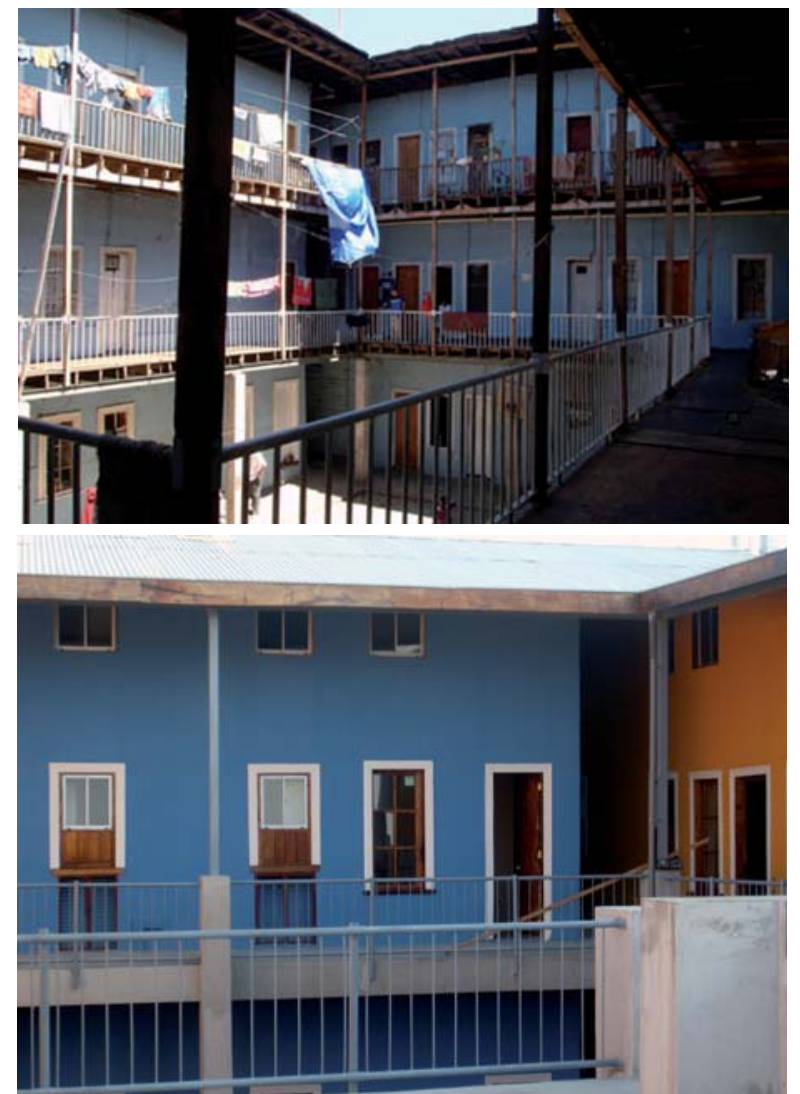

> Figura 2. Rehabilitación de la Unión Obrera. Antes y después. Fotografías M. Soto, 2008 
a ver los actos, lo que hacen con los cabros chicos, cuando me llaman, pero encuentro un poco que le hace falta más política, ahí no se habla de política, ahí no se lleva al debate, no se discute. Falta la contingencia, y vamos formando un grupo pequeño de... ya si hay que ir a apoyar a la calle, vamos en representación del Santa Ana apoyando en la caIle... como que viven ahí encerrados en sus cuestiones y a mí eso no me calza... me falta más política y preparación en la cuestión política (...) Si siguen en esa onda, de seguir con los proyectitos y moviéndole la cola a la autoridad allá, a la autoridad acá, van a terminar replicando el sistema de la Concerta y de la Nueva Mayoría...y yo todavía ahí no veo que haya autogestión, que dicen que es un sistema autogestionado, comunitario, todavía no. Yo sé que eso no debe ser nada de fácil hacerlo, pero puede estar embromando mucho ésta, porque me da la impresión de que hay una cantidad grande de gente que le gusta esto de los préstamos y estar pidiendo y estar bien con las autoridades (María Eugenia Velasco, entrevista del 15.03.2014).

El Espacio Santa Ana es una comunidad donde se percibe una yuxtaposición de capas sociales, y donde lo sedimentario y transitorio cohabitan, permitiendo una reafiliación identitaria basada en una interpretación de lo Comunitario. Es desde ésta dinámica donde la noción de un compartir transitorio o efímero va definiendo las relaciones e interacciones al interior del Espacio Santa Ana, desde donde se abordan acciones precisas sobre el territorio.

Por ejemplo en julio de 2013 la ex capilla sufre el derrumbe de uno de sus muros de adobe, una situación concreta que produce nuevos desafíos en el grupo de actores del lugar, los llamados "santaninos". En el momento que empezamos a visitar el lugar, una serie de miembros y voluntarios estaban organizando las labores necesarias para la autoconstrucción de ladrillos de adobe. Una actividad que invitaba a la comunidad del Cerro Cordillera, respondiendo a una reapropiación comunitaria del territorio.

Se levantaron diversas jornadas para la autoconstrucción de ladrillos de adobe con características de ser antisísmicos, para todo tipo de temblores. Promocionando que era una experiencia relevante para reactivar espacios de encuentro y acción comunitaria.

PON TU GRANITO DE TIERRA, COMPARTE TU FUERZA PARA CUMPLIR CON EL PROPÓSITO COMÚN: PARA EL MURO QUE SE DERRUMBÓ EL INVIERNO PASADO. YA SABES, YA ESTAMOS TRABAJANDO, ESCUCHANDO BUENA MÚSICA. HOY SOMOS OCHO MAÑANA PODEMOS SER MÁS!!! (Espacio Santa Ana, (2014) Facebook)

Antes de comenzar las labores de autoconstrucción de adobes fijada para el 10 de febrero 2014, miembros de la comisión de comunicación colocaron un afiche: Se convoca a la participación de todos y todas a la recuperación colectiva de este espacio comunitario y encuentro territorial. Para los miembros del Espacio Santa Ana la vinculación con el territorio y su comunidad responde a prácticas ya inscritas en el Cerro Cordillera desde las acciones ya emprendidas por el TAC. Desde donde la recuperación de la ex capilla, desde una acción colectiva/comunitaria representa también una necesidad de conservar el patrimonio material, y así poder abrirlo al público como un espacio de encuentro; donde se puedan realizar servicios culturales, deportivos, artísticos y así ir fomentando la vida social en comunidad del cerro.
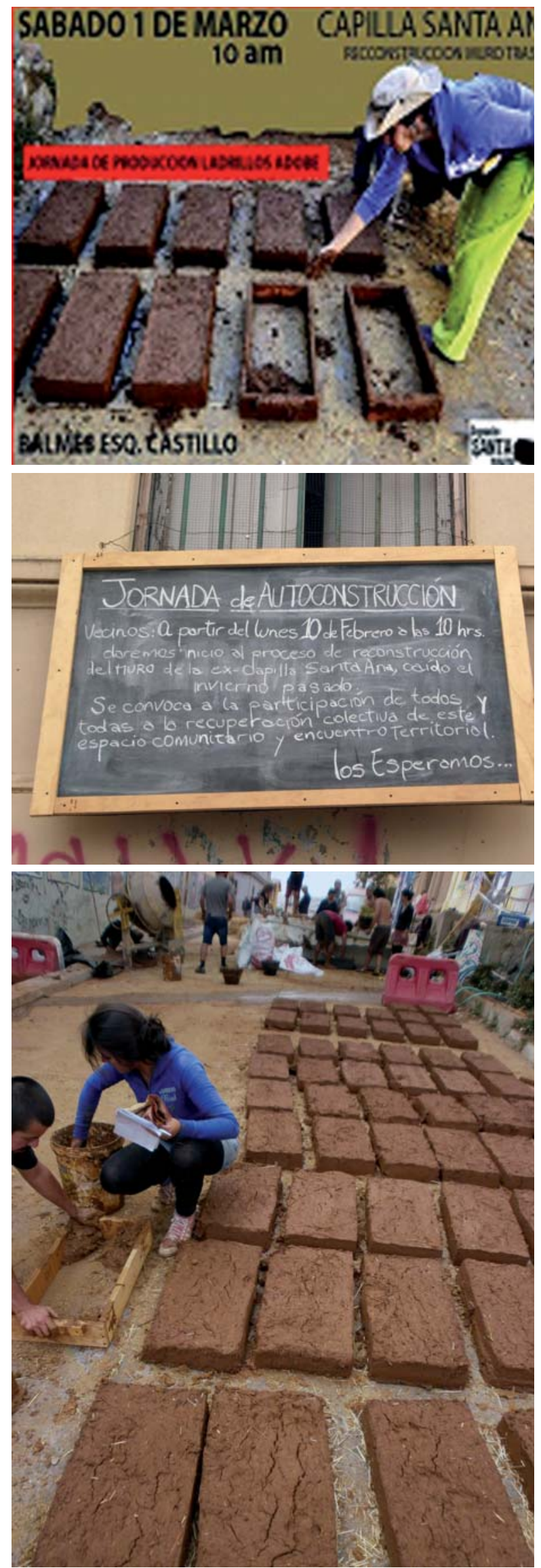

> Figura 3. Jornada de autoconstrucción de adobes en el Espacio Santa Ana. Fotografías M. Soto, 2014 
Como se hace siempre la escuela de invierno y de verano, que son las dos actividades bien grandes que tiene el TAC, que son durante las vacaciones de los niños. Donde hay hartos niñitos... me dijo: oye vamos a esto..., bueno, cuento corto, fui y participé, buena onda, y salió como una actividad simpática, en ese tiempo, en... las vacaciones de invierno de mi primer año acá. Después en verano me dijo: oye vamos de nuevo, y la cuestión... Bueno, después de ese verano en adelante, empecé a involucrarme más con Cordillera, con las actividades que se siguen acá, en ese tiempo se estaba trabajando en la reconstrucción de Santa Ana, sólo de la capilla, no estaba este lugar acá... eh, me acuerdo que en ese tiempo había, se estaba haciendo un ciclo de teatro (Charly, miembro del Espacio Santa Ana, entrevista del 10.03.2014).

El adobe es una pieza similar a un ladrillo, fabricado con barro y paja, material común en la fabricación de casas en la antigüedad lo que convierte al cerro Cordillera en un cerro antiguo e histórico. Incluso una de las organizaciones comunitarias, el Espacio Santa Ana, le dio un valor simbólico al adobe ya que se fabricaron piezas de este material para la reconstrucción de la ex capilla Santa Ana. Esta actividad fue difundida hacia los vecinos lo que se expresa en un poste de calle Jiménez donde hay un segundo cartel que dice vecinos/as en febrero comenzamos con la recuperación del muro de la ex capilla Espacio Santa Ana iparticipa!

Este entorno de interacción se estructura en función de prácticas de reapropiación que conforman un espacio comunitario organizado en comisiones para cumplir los objetivos de formación y seguridad del lugar. En el Espacio Santa Ana se realizan diversas actividades todos los días de la semana, actualmente se está llevando a cabo un proyecto de un jardín infantil "La madriguera", por lo que es común ver a distintas horas del día entrar y salir niños y niñas con sus respectivos padres y/o cuidadores del recinto, éstos últimos en su mayoría son jóvenes. Proyectos y actividades que responden a prácticas culturales y a formas sociales inscritas en el territorio que se articulan, a modo de reapropiación, desde el trabajo realizado por el TAC.

Si yo creo que en general, no sé si será responsabilidad del TAC que se genere el cambio en la persona pero sí, hay un aporte creo yo. Lo que se desarrolla es eso, el tema educacional y el tema de la recuperación de espacios, partiendo desde lo que fue este espacio de quebrada que era un basural que se transformó en un huerto, un anfiteatro, ese era como el principio del TAC; ese principio se empieza a reproducir en otros espacios... En el caso de la biblioteca Gutemberg, que puntualmente es un espacio que es de la población, que es la población Gutenberg, que tiene todo su cuento y su historia atrás, y la casona misma también tiene todo su cuento, que es la casona que se da, por una razón de que ya llevaban tiempo trabajando al servicio del TAC... A cualquier organización le va a costar tener dos espacios de trabajo al mismo tiempo, entonces lo que se da, el vínculo, el trato entre la población Gutenberg y el TAC, es ese, ese es el vínculo que hace que llegue gente a trabajar a la biblioteca, que como el dato que se dio de la población al TAC, como organización reconocida en el Cerro, que se hiciera cargo de la administración de la casona. De ahí llevan voluntarios al TAC de distintas partes, yo por mi parte llegue del TAC a la biblioteca. Hay vínculos, a veces somos los mismos, las mismas personas pero los es- pacios son distintos, y los espacios tienen distintos enfoques, aun cuando el enfoque general sigue siendo el mismo, la autogestión, el trabajo comunitario, la recuperación de espacios, etc... (Charly, miembro del Espacio Santa Ana, entrevista del 10.03.2014).

El trabajo comunitario por tanto, debe ser diferenciado de otras prácticas que no tienen como eje la organización de la población. El trabajo comunitario se sitúa sobre el concepto de autogestión y lo importante es comprender que sin un "sujeto colectivo autónomo" no se podría hablar de trabajo comunitario ni de acción colectiva.

\section{CONCLUSIÓN}

Desde las etnografías realizadas en la parte alta del Cerro Cordillera, hemos podido analizar una dinámica de continuidad en función de prácticas de reapropiación de un espacio particular de Valparaíso. Dinámicas socio-culturales que nos permiten incorporar el concepto de entorno de interacción como concepto operatorio que permite profundizar los conceptos de lugar (Augé, 1992) o de espacio social (Bourdieu, 1984), para dar forma a nuevos métodos de análisis del territorio que ayudan a estudiar las prácticas que se están ejerciendo y que a modo de una lucha por el reconocimiento son capaces de dar señas de la producción de un Nosotros cultural singular del Cordillera.

\section{BIBLIOGRAFÍA}

AUGÉ, Marc (1992) Non-lieux. Introduction à une anthropologie de la surmodernité. París: Seuil.

BENAVIDES, Juan; PIZZI, Marcela; VALENZUELA, María Paz (1994) Ciudades y Arquitectura portuaria. Valparaíso: Editorial Universitaria.

BLANC, Maurice (2009) La transaction sociale: genèse et fécondité heuristique. Pensée Plurielle (Bruselas), № 20, pp. 25-36.

BOURDIEU, Pierre (1993) Effets de lieu. En Bourdieu, P. (dir.) La Misère du Monde, París: Seuil.

(1984). Espace social et genèse des clases. Actes de la recherche en sciences sociales (París), Vol. LII-LIII, pp. 3-14.

BOUTINET, Jean-Pierre (2005@1990) Anthropologie du projet. París: PUF.

BOUVIER, Pierre (2011) De la socioanthropologie. París: Galilée.

CALDERóN, Alfonso (1986) Memorial de Valparaíso. Valparaíso: Ediciones Universitarias de Valparaíso.

CANDAU, Joel (1996) Anthropologie de la mémoire. París: PUF.

CASTEL, Robert (1991) De l'indigence à l'exclusion, la désaffiliation. Précarité du travail et vulnérabilité relationnelle. En Donzelot, J. (dir.) Face à l'exclusion. Le modèle français. París:Esprit, pp. 137-168.

CASTILLO, Oscar (2005) La ciudad-puerto de Valparaíso como patrimonio arquitectónico e histórico-cultural de la humanidad. Revista archivum año IV, N 5, pp. 27-39.

CAVIERES, Eduardo (1986) Urbanización y cambio social: comerciantes y artesanos de Valparaíso en la segunda mitad del siglo XIX. Monografías históricas, Vol. I, Valparaíso 15361986, Universidad Católica de Valparaíso, pp. 57-76. 
CEFAÏ, Daniel (dir.) (2003) L'enquête de terrain. París: La Découverte.

CLIFFORD, James (1995) Sobre la autoridad etnográfica. En Clifford, J. Dilemas de la Cultura. Antropología, literatura y arte en la perspectiva postmoderna. Barcelona: Gedisa, pp. 39-77.

(1995) Identidad en Mashpee. En Clifford, J. Dilemas de la Cultura. Antropología, literatura y arte en la perspectiva postmoderna. Barcelona: Gedisa, pp. 327-406.

CORREA, Pilar (2013) Nuevas relaciones Estado - Organizaciones de la sociedad civil. Aprendizajes del Taller de Acción Comunitaria. TAC, Cordillera. http://www.tesis.uchile.cl/ handle/2250/113522 (Consultado el 20.05.2014).

COUYOUMDJIAN, Juan Ricardo (2000) El alto comercio de Valparaíso y las grandes casas extranjeras, 1880-1930. Una aproximación. Historia (Santiago) Vol. 33, pp. 63-99.

CRUZ, Alberto (2003). Revisitar. En Montecino, S. (dir.) Revisitando Chile. Identidades, mitos e historias. Santiago: Cuadernos Bicentenario, pp. 217- 220.

DEBARBIEUX, Bernard (1996). Le lieu, fragment et symbole du territoire. Espace et Société (París), № 82-83, pp. 13-35.

DESCOLA, Philippe (2005) Par-delà nature et culture. París: Gallimard.

DOUGLAS, Mary (2005@1966) De la souillure: Essais sur les notions de pollution et de tabou. París: La Découverte.

FERRADA, Mario y JIMÉNEZ, Cecilia (2007) La primera vivienda social en Valparaíso. Fines siglo XIX-inicios siglo XX. En Castillo, M. J. e Hidalgo, R. (dir.) Cien años de política de vivienda en Chile. Santiago: Universidad Andrés Bello.

GEERTZ, Clifford (2003) La description dense. Vers une théorie interprétative de la culture. En Cefaï, D (dir.) L'enquête de terrain, París: La Découverte, pp. 208-233.

GODELIER, Maurice (1984) L'idéel et le matériel. Pensée, économies, sociétés. París: Fayard.

GRAFMEYER, Yves (1999) La coexistence en milieu urbain: échanges, conflits, transactions. Recherches Sociologiques (Bruselas), Vol. XXX, N 1, pp. 157-176.

GRAVANO, Ariel (2003) Antropología de lo Barrial. Estudios sobre la producción de la vida urbana. Buenos Aires: Editorial Espacio.

HANNERZ, Ulf (1983) Explorer la ville: éléments d'anthropologie urbaine. París: Minuit.

HONNETH, Axel (2002@1992) La lutte pour la reconnaissance. París: Cerf.

LAPLANTINE, François (1996) La description ethnographique. París: Nathan université, coll. "128".

LEDRUT, Raymond (1976) L'Espace en question ou le nouveau monde urbain. París: Anthropos.

LEFEBVRE, Henri (2000 @1974) La production de l'espace. París: Anthropos.

LINDÓN, Alicia (2007) La ciudad y la vida urbana a través de los imaginarios urbanos. EURE (Santiago), Vol.33, № 99, pp. 7-16.

MATAS, Juan (2003) Des frontières côté sud. Revue des sciences sociales (Estrasburgo), № 31, pp. 26-33.
PINTO, Aníbal (1959) Chile, un caso de desarrollo frustrado. Santiago: Edición Universitaria.

REMY, Jean (1995) La forme et l'auto-organisation du social: la méthodologie implicite de Georg Simmel. París: L'Harmattan.

(1992) La vie quotidienne et les transactions sociales: perspectives micro ou macro-sociologiques. En Blanc, M. (dir.) Pour une sociologie de la transaction sociale. Paris: L'Harmattan, pp. 83-111.

REMY, Jean; VOYÉ, Liliane et SERVAIS, Emile (1991@1978) Produire ou reproduire? une sociologie de la vie quotidienne. Vol. 1 "Conflits et transaction sociale». Bruxelles: De Boeck.

REYNAUD, Jean-Daniel (1989) Les règles du jeu. L'action collective et la régulation sociale. París: Armand Colin.

(1979) Conflit et régulation sociale. Esquisse d'une théorie de la régulation conjointe. Revue française de sociologie. Vol. XX, pp. 367-376.

RICCEUR, Paul( 2002) La lutte pour la reconnaissance et l'économie du don. Conférence à la Première Journée de la philosophie à l'Unesco, 21 novembre 2002.

SÁEZ, Leopoldo (2001) Valparaíso. Lugares, nombres y personajes, siglos XVI-XXI. Valparaíso: Puntángeles, Universidad de Playa Ancha y USACH.

SALAZAR, Gabriel (1985) Labradores, peones y proletarios. Formación y crisis de la sociedad popular chilena del siglo XIX. Santiago: Ediciones Sur.

SCHUTZ, Alfred (1972) Fenomenología del Mundo Social. Buenos Aires: Paidós.

SIMMEL, Georg (1996@1908) Secret et sociétés secrètes. París: Circé/poche.

SOTO, Maximiliano (2014a) Del uso laboral al uso residencial: análisis socio-semiótico de la reconversión de un taller de manufactura, La Igualdad. Revista Llámpara Patrimonio Industrial (Portoalegre), $N^{\circ}$ 6, pp. 16-27.

Les paradoxes de la mise en valeur des ascensores (funiculaires) de Valparaiso: le conflit entre patrimonialisation matérielle et immatérielle. L'Homme et la Société, N 191 , pp. 139-156.

URBINA, Ximena (2002) Los conventillos de Valparaíso 18801920, fisonomía y percepción de una vivienda popular urbana. Valparaíso: Ediciones Universitarias de Valparaíso.

NOTAS

1 Simmel concentra su análisis en las metrópolis, centrando el análisis sociológico de la ciudad en la definición de formas inter-psyches. La forma aparece como un modo de estructuración de lo social. La forma está dotada de propiedades estructurales en la medida donde ella es capaz de articular tensiones entre dos polos en competencia (Remy, 1995:149).

2 Las etnografías se apoyan en una pauta de observación con el fin de establecer los elementos a identificar y describir al interior y exterior de los tres lugares señalados (Laplantine, 1996). Observaciones que serán complementadas con un registro fo- 
tográfico de las principales actividades poniendo atención en los locatarios y en sus quehaceres cotidianos.

3 Este esquema se inspira en los trabajos realizados por Axel Honneth: los individuos de baja estima son obligados a "luchar por el reconocimiento" (Honneth, 2002).

Dentro del alto comercio de Valparaíso se pueden contar un total de 233 casas importadoras, y 97 gerencias comerciales, además de 36 almacenes mayoristas, 84 bodegas públicas, 62 almacenes de abarrotes y otros, 48 escritorios comerciales, 18 casas de consignaciones y 6 depósitos de calzado (Juan Ugarte, Valparaíso 1536-1910, Recopilación Histórica, Comercial y Social, in Couyoumdjian, 2000:66). 